\title{
Editorial: Physical Review A: An evolving and leading-edge journal with a rich history
}

Throughout 2018, APS has been celebrating the 125th anniversary of The Physical Review. In comparison, Physical Review A-which was born under the heading "General Physics" when the original publication was split into four parts in 1970 - is a young journal. Even so, we can already look back on an almost-50year history. Since its founding, the journal has continuously grown in size and reach. While the first issue, in January 1970, had 33 articles, we now publish more than 200 articles each month. And although 28 of those first 33 articles had authors only from the US, we are now truly international: in 2017, $84 \%$ of the manuscripts submitted to us came from abroad.

Grounded in the tradition of The Physical Review, the mission of Physical Review A has always been to publish excellent physics within the scope of the journal, and our approach was captured in a formal statement adopted by the APS Council in 1995: "It is the policy of the American Physical Society that the Physical Review accept for publication those manuscripts that significantly advance physics and have been found to be scientifically sound, important to the field, and in satisfactory form. The Society will implement this policy as fairly and efficiently as possible and without regard to national boundaries."

This is a promise to our authors that they will be treated as fairly as possible and that we do not discriminate on the basis of nationality, gender, religion, political views, or other aspects that are not part of the scientific assessment of their work. And it is a promise to our readers that, in addition to being free from obvious errors, the papers they see published in Physical Review A have been judged by their peers to represent substantial advances. These guiding principles have not changed in decades and form the anchor for the journal.

Among the more than 77,000 papers Physical Review A has published, there are many jewels that would make any journal proud. The most highly cited, a paper on density-functional theory by A.D. Becke, has received more than 22,000 citations, making it the 5th-most-cited paper in the 125-year history of the Physical Review family. Other highlights include seminal papers by Loss and DiVincenzo on quantum computation with quantum dots, by Bennett et al. on quantum error correction, and by Allen et al. on orbital angular momentum of light, to name just a few that have amassed over 2,000 citations each. And over the last twenty-five years, eleven Nobel prizes have been awarded in areas directly related to work published in Physical Review A, and all of the recipients have published some of their work in our journal.

While we are proud of this rich history, it does not mean that we should rest on our laurels; on the contrary, the editors are motivated to continue to work hard so that Physical Review A will remain the journal of choice in the areas of physics that we cover. In order to achieve this, the journal has always evolved, and continues to do so, to adapt to the changing needs of the community.

For example, a significant change, rooted in the ever-increasing size of the journal, was to begin highlighting a small number of papers that the editors feel deserve special attention due to their particular interest, significance, or clarity. Of course, we feel that all the papers published in Physical ReviewA deserve attention, but the sheer amount of available information has made it increasingly difficult for readers to follow developments that are somewhat outside their own main areas of specialization. In order to help our readers navigate this information, we introduced Editors' Suggestions in August 2013. Now, five years later, we are about to publish our 500th Suggestion.

Even more important are the changes to the content itself-the scope of the journal. When the journal became too large and seemed to lack focus, Physical Review E was split off from Physical Review A in 
January 1993, and Physical Review A concentrated on atomic, molecular, and optical physics. Being one of the first journals to fully embrace quantum information as a new research field, we created a separate section entitled Quantum Information as early as January 1998.

In the ensuing two decades, this section has grown to be the second-largest section of the journal, and now contains about twenty percent of all the papers published in Physical Review A. In recognition of this, we have recently added "Quantum Information" to our formal statement of coverage, to both recognize that quantum information has evolved into its own subfield of physics, and to make it clear that Physical Review $A$ views itself as the home for high-quality papers in this area.

Along similar lines, we have very recently responded to the needs of another community by renaming one of the sections of the journal. While APS has had a Topical Group on Precision Measurement and Fundamental Constants since 1987, the field has recently seen a surge, and more and more papers in this area are being published, many of them in Physical Review A. Thus in July 2018 we changed the title of the "Atomic and molecular structure and dynamics" section to "Atomic and molecular structure and dynamics; high-precision measurements," to signal that this community clearly has a home in our journal.

For almost fifty years, Physical Review A has been an indispensable part of the AMO community and (for a somewhat shorter time) the quantum information community. The journal has grown with its community, and has, in turn, facilitated and enabled the growth of the community itself. While we feel that we have much to be proud of, we will not be complacent. We will continue to listen to our readers, authors, and referees, and adapt, within the framework of our mission, to their needs.

Thomas Pattard is Managing Editor of Physical Review A and has been with the journal since 2006. Gordon Drake has been Lead Editor of Physical Review A since 2006. He is a former Head of the Department of Physics at the University of Windsor, a Fellow of the Royal Society of Canada, and an APS Fellow.

[This information also appears in the August/September 2018 issue of APS News aps.org/publications/ APSnews.]

Thomas Pattard Gordon Drake

Published 4 September 2018

DOI: 10.1103/PhysRevA.98.030001 